\title{
ON FUNCTIONS HAVING SUBHARMONIC LOGARITHMS
}

\author{
MAXWELL O. READE
}

Saks has shown that if $f(x, y)$ is subharmonic in a domain $G$ and if $\mu(e)$ is the completely additive, non-negative function of Borel sets associated with $f(x, y)$, then

$$
\lim _{\rho \rightarrow 0} \frac{8}{\rho^{2}}\left[\frac{1}{\pi \rho^{2}} \iint f(x+\xi, y+\eta) d \xi d \eta-f(x, y)\right]=D_{s} \mu(x, y),
$$

and

$$
\lim _{\rho \rightarrow 0} \frac{4}{\rho^{2}}\left[\frac{1}{2 \pi \rho} \int f(x+\xi, y+\eta) d s-f(x, y)\right] \quad=D_{s} \mu(x, y)
$$

hold almost everywhere in $G$ [7]. ${ }^{1}$ Here the first integral is extended over all $(\xi, \eta)$ such that $\xi^{2}+\eta^{2}<\rho^{2}$, the second integral is extended over all $(\xi, \eta)$ such that $\xi^{2}+\eta^{2}=\rho^{2}$, and $D_{s} \mu(x, y)$ is the symmetric derivative of $\mu(e)$ at $(x, y)$.

The main result of this paper is an analogue of Saks' result for continuous functions having subharmonic logarithms. For such functions $f(x, y)$, it is shown that if $\sigma(e)$ is the completely additive, non-negative function of Borel sets associated with $\log f(x, y)$, then

$$
\begin{array}{r}
\lim _{\rho \rightarrow 0} \frac{4}{\rho^{2}}\left\{\left(\frac{1}{2 \pi \rho} \int f(x+\xi, y+\eta) d s\right)^{2}-\frac{1}{\pi \rho^{2}} \iint f^{2}(x+\xi, y+\eta) d \xi d \eta\right\} \\
=f^{2}(x, y) D_{s} \sigma(x, y)
\end{array}
$$

holds almost everywhere in $G$.

Let $G$ denote a domain (non-null connected open set) in the $x, y$ plane, $D(x, y ; \rho)$ the open circular disc with center at $(x, y)$ and radius $\rho$, and $C(x, y ; \rho)$ the boundary of $D(x, y ; \rho)$. If $f(x, y)$ is continuous in $G$, then $f(x, y)$ is said to be subharmonic in $G$ if and only if

$$
f(x, y) \leqq A(f ; x, y ; \rho) \equiv \frac{1}{\pi \rho^{2}} \iint_{D(x, y ; \rho)} f(\xi, \eta) d \xi d \eta
$$

holds for each $D(x, y ; \rho)$ in $G$ [4]. It is well known that (1) can be replaced by either [4]

Presented to the Society, April 28, 1944, under the title On functions of class $P L$; received by the editors May $1,1946$.

${ }^{1}$ Numbers in brackets refer to the bibliography at the end of the paper. 


$$
f(x, y) \leqq L(f ; x, y ; \rho) \equiv \frac{1}{2 \pi \rho} \int_{C(x, y ; \rho)} f(\xi, \eta) d s,
$$

or

$$
A(f ; x, y ; \rho) \leqq L(f ; x, y ; \rho) .
$$

An important subclass of the class of functions subharmonic in $G$ consists of those functions having subharmonic logarithms. These functions, studied by Beckenbach and Radó [1], are defined as follows. A function is said to be of class $P L$ in $G$ if and only if (i) $f(x, y) \geqq 0$, (ii) $f(x, y) \not \equiv 0$, (iii) $\log f(x, y)$ is subharmonic in $G$. It is fundamental in the theory of functions of class $P L$ in $G$ that $f(x, y)$ is of class $P L$ in $G$ if and only if

$$
A\left(f^{2} ; x, y ; \rho\right) \leqq[L(f ; x, y ; \rho)]^{2}
$$

holds for each $D(x, y ; \rho)$ in $G[1]$.

If $f(x, y)$ has continuous partial derivatives of the second order in $G$, then $f(x, y)$ is subharmonic in $G$ if and only if $\Delta f(x, y) \geqq 0$ in $G$, and $f(x, y)$ is of class $P L$ in $G$ if and only if

$$
f^{2} \Delta \log f \equiv f \Delta f-\left(\frac{\partial f}{\partial x}\right)^{2}-\left(\frac{\partial f}{\partial y}\right)^{2} \geqq 0
$$

in $G[4]$. Here $\Delta$ is the Laplace operator

$$
\Delta \equiv \frac{\partial^{2}}{\partial x^{2}}+\frac{\partial^{2}}{\partial y^{2}} .
$$

If $f(x, y)$ is subharmonic in $G$, then Riesz [6] has shown that there exists a unique, completely additive, non-negative function $\mu(e)$ of Borel sets $e$ (for which the closure $\bar{e} \subset G$ ) with the following property. If $D$ is a subdomain of $G$, such that $\bar{D} \subset G$, then $f(x, y)$ has the representation

$$
f(x, y) \equiv f(P)=-\frac{1}{2 \pi} \iint_{D} \log \frac{1}{P Q} d \mu\left(e_{Q}\right)+H(P), \quad P \in D,
$$

where $P Q=\left((x-\xi)^{2}+(y-\eta)^{2}\right)^{1 / 2}, H(P)$ is harmonic in $D$, and where the integral is a Stieltjes-Radon integral [8].

Since the density of $\mu(e)$ at $(x, y)$ is defined by [8]

$$
D_{s} \mu(x, y) \equiv \lim _{\rho \rightarrow 0} \frac{\mu[D(x, y ; \rho)]}{\pi \rho^{2}}
$$

(which is known to exist almost everywhere [8]), then Saks' result 
may be stated as follows. If $f(x, y)$ is subharmonic in $G$, and if $\mu(e)$ is the set function used in (3), then

$$
\begin{aligned}
\lim _{\rho \rightarrow 0} \frac{8}{\rho^{2}}[A & (f ; x, y ; \rho)-f(x, y)] \\
& =\lim _{\rho \rightarrow 0} \frac{4}{\rho^{2}}[L(f ; x, y ; \rho)-f(x, y)]=D_{s} \mu(x, y)
\end{aligned}
$$

holds almost everywhere in $G$. Saks' proof of (5) depends upon the representation (3) for $f(x, y)$.

If $f(x, y) \geqq 0$ is continuous and subharmonic in $G$, then $f^{2}(x, y)$ is continuous and subharmonic in $G$ [4]. Hence by the "representation theorem" of Riesz, noted above, there exist unique, completely additive, non-negative set functions $\mu(e)$ and $\nu(e)$, for $\bar{e} \subset G$, associated with $f(x, y)$ and $f^{2}(x, y)$, respectively. Then the following lemmas hold.

LEMMA 1.

$$
\begin{aligned}
\lim _{\rho \rightarrow 0} \frac{1}{\rho^{2}}\left[L^{2}(f ; x, y ; \rho)\right. & \left.-A\left(f^{2} ; x, y ; \rho\right)\right] \\
& =\frac{f(x, y) D_{s} \mu(x, y)}{2}-\frac{D_{s} \nu(x, y)}{8}
\end{aligned}
$$

holds almost everywhere in $G$.

Proof. It is well known [4] that $L(f ; x, y ; \rho) \rightarrow f(x, y)$ and $A\left(f^{2} ; x, y ; \rho\right) \rightarrow f^{2}(x, y)$ on $\bar{e} \subset D$, as $\rho \rightarrow 0$. The relation (6) now follows from (5) and the identity

$$
\begin{aligned}
& \frac{L^{2}(f ; x, y ; \rho)-A\left(f^{2} ; x, y ; \rho\right)}{\rho^{2}} \\
& \quad=[L(f ; x, y ; \rho)+f(x, y)]\left[\frac{L(f ; x, y ; \rho)-f(x, y)]}{\rho^{2}}\right] \\
& \quad-\frac{A\left(f^{2} ; x, y ; \rho\right)-f^{2}(x, y)}{\rho^{2}} .
\end{aligned}
$$

LemMa 2. If $e$ is a Borel set, $\bar{e} \subset G$, then

$$
\nu(e)=2 \iint_{e} f(P) d \mu\left(e_{P}\right)+2 \iint_{e}\left[\left(\frac{\partial f}{\partial x}\right)^{2}+\left(\frac{\partial f}{\partial y}\right)^{2}\right] d x d y .
$$

Proof. Let $D$ be a subdomain of $G$, such that $\bar{D} \subset G$. It follows from the proofs of the representation (3) for subharmonic functions, given by Evans [2] and Riesz [6], that $\mu(e)$ and $\nu(e)$ may be obtained as follows. If iterated averages of $f(x, y)$ are defined as 
$A_{2}(f ; x, y ; \rho) \equiv A(A ; x, y ; \rho), \quad A_{3}(f ; x, y ; \rho) \equiv A\left(A_{2} ; x, y ; \rho\right)$, then there exists a sequence $\left\{\rho_{n}\right\} \searrow 0$, as $n \rightarrow \infty$, such that the set functions

$$
\begin{aligned}
\mu_{n}(e) & \equiv \iint_{e} \Delta A_{3}\left(f ; x, y ; \rho_{n}\right) d x d y, \\
\nu_{n}(e) & \equiv \iint_{e} \Delta\left[A_{3}\left(f ; x, y ; \rho_{n}\right)\right]^{2} d x d y,
\end{aligned}
$$

converge to $\mu(e)$ and $\nu(e)$, respectively; that is,

$$
\lim _{n \rightarrow \infty} \mu_{n}(e)=\mu(e),
$$

if $e$ is open and $\mu$-regular (that is, $\mu(\bar{e}-e)=0$ ) and

$$
\lim _{n \rightarrow \infty} \nu_{n}(e)=\nu(e),
$$

if $e$ is open and $\nu$-regular (that is, $\nu(\bar{e}-e)=0$ ).

Now if $R$ is an oriented rectangle in $D$, and if $R$ is both $\mu$ - and $\nu$-regular, and if the substitution

$$
A_{3}\left(f ; x, y ; \rho_{n}\right) \equiv \mathfrak{A}_{n}(x, y)
$$

is made, then it follows from (8) and (9) that

$$
\begin{aligned}
\nu(R)= & \lim _{n \rightarrow \infty} 2 \iint_{R} \mathfrak{A}_{n}(P) d \mu_{n}\left(e_{P}\right) \\
& +\lim _{n \rightarrow \infty} 2 \iint_{R}\left[\left(\frac{\partial \mathfrak{A}_{n}}{\partial x}\right)^{2}+\left(\frac{\partial \mathfrak{A}_{n}}{\partial y}\right)^{2}\right] d x d y
\end{aligned}
$$

holds. However, Frostman has shown [3] that if $R$ is $\mu$-regular, then

$$
\lim _{n \rightarrow \infty} \iint_{R} \mathfrak{A}_{n}(P) d \mu_{n}\left(e_{P}\right)=\iint_{R} f(P) d \mu\left(e_{P}\right),
$$

and Evans has shown [2] that

$$
\lim _{n \rightarrow \infty} \iint_{R}\left[\left(\frac{\partial \mathfrak{A}_{n}}{\partial x}\right)^{2}+\left(\frac{\partial \mathfrak{A}_{n}}{\partial y}\right)^{2}\right] d x d y=\iint_{R}\left[\left(\frac{\partial f}{\partial x}\right)^{2}+\left(\frac{\partial f}{\partial y}\right)^{2}\right] d x d y
$$

so that (10) may be written

$$
\nu(R)=2 \iint_{R} f(P) d \mu\left(e_{P}\right)+2 \iint_{R}\left[\left(\frac{\partial f}{\partial x}\right)^{2}+\left(\frac{\partial f}{\partial y}\right)^{2}\right] d x d y .
$$


Now each open oriented rectangle $R$ in $D$ is the point set limit of a monotone increasing sequence $\left\{R_{n}\right\}$ of open $\mu$ - and $\nu$-regular rectangles, such that [5]

$$
\begin{aligned}
& \lim _{n \rightarrow \infty} \mu\left(R_{n}\right)=\mu(R), \\
& \lim _{n \rightarrow \infty} \nu\left(R_{n}\right)=\nu(R) .
\end{aligned}
$$

Hence it follows from (11), (12) and (13) that (11) must hold for all open oriented rectangles $R$ in $D$. By a familiar argument used in the theory of set functions $[\mathbf{5}, \mathbf{7}]$, it follows that (7) holds for all Borel sets in $D$.

Since $D$ was an arbitrary subdomain of $G$, the lemma now follows.

Corollary.

$$
D_{s} \nu(x, y)=2\left[f(x, y) D_{s} \mu(x, y)+\left(\frac{\partial f}{\partial x}\right)^{2}+\left(\frac{\partial f}{\partial y}\right)^{2}\right]
$$

almost everywhere in $G$.

Proof. The relation (14) follows from (4), (7) and the classic theorem due to Lebesgue on the derivation of integrals [8].

TheOREM 1.

$$
\begin{aligned}
\lim _{\rho \rightarrow 0} \frac{4}{\rho^{2}}\left[L^{2}(f ; x, y ; \rho)\right. & \left.-A\left(f^{2} ; x, y ; \rho\right)\right] \\
= & f(x, y) D_{\imath} \mu(x, y)-\left(\frac{\partial f}{\partial x}\right)^{2}-\left(\frac{\partial f}{\partial y}\right)^{2}
\end{aligned}
$$

almost everywhere in $G$.

Proof. (15) follows from (6) and (14).

In the following, it is assumed that $f(x, y)$ is also of class $P L$ in $G$. Hence $f(x, y) \equiv \exp u(x, y)$, where $u(x, y)$ is continuous and subharmonic in $G$, with associated set function called $\sigma(e)$.

Lemma 3. If $e$ is a Borel set, $\bar{e} \subset G$, then

$$
\begin{aligned}
\mu(e)= & \iint_{e} \exp \mu(P) d \sigma\left(e_{P}\right) \\
& +\iint_{e}\left[\left(\frac{\partial u}{\partial x}\right)^{2}+\left(\frac{\partial u}{\partial y}\right)^{2}\right] \exp u(x, y) d x d y .
\end{aligned}
$$


Proof. It is inherent in the proof of (3) given by Evans [2] and Riesz [6], that $\mu(e)$ and $\sigma(e)$ may be found as follows. If the definition

$$
A_{3}\left(u ; x, y ; \rho_{n}\right) \equiv a_{n}(x, y)
$$

is made, then there exists a sequence $\left\{\rho_{n}\right\} \searrow 0$, as $n \rightarrow \infty$, such that

$$
\mu_{n}^{*}(e) \equiv \iint_{\theta} \Delta\left[\exp a_{n}(x, y)\right] d x d y
$$

and

$$
\sigma_{n}(e) \equiv \iint_{0} \Delta a_{n}(x, y) d x d y
$$

converge to $\mu(e)$ and $\sigma(e)$, respectively; that is

$$
\lim _{n \rightarrow \infty} \mu_{n}^{*}(e)=\mu(e)
$$

for each open $\mu$-regular set $e$, and

$$
\lim _{n \rightarrow \infty} \sigma_{n}(e)=\sigma(e)
$$

for each open $\sigma$-regular set $e$.

Now an argument similar to that used in the proof of Lemma 2 shows that (16) follows from (17)-(20).

Corollary.

$$
\begin{aligned}
D_{s} \mu(x, y)= & \exp u(x, y) \cdot D_{s} \sigma(x, y) \\
+ & {\left[\left(\frac{\partial u}{\partial x}\right)^{2}+\left(\frac{\partial u}{\partial y}\right)^{2}\right] \exp u(x, y) }
\end{aligned}
$$

almost everywhere in $G$.

Proof. The corollary follows at once from (4), (16) and the theorem of Lebesgue on the derivation of integrals.

THEOREM 2.

(22) $\lim _{\rho \rightarrow 0} \frac{4}{\rho^{2}}\left[L^{2}(f ; x, y ; \rho)-A\left(f^{2} ; x, y ; \rho\right)\right]=f^{2}(x, y) D_{s} \sigma(x, y)$

almost everywhere in $G$.

Proof. The theorem, which is an analogue of Saks' result (5), follows at once from (15) and (21). 
The relations (5), (6), (15) and (22) are examples of "generalized Laplacians" [7, 9]. For example, if $f(x, y)$ is sufficiently smooth in $G$, then (22) yields

$$
\lim _{\rho \rightarrow 0} \frac{4}{\rho^{2}}\left[L^{2}(f ; x, y ; \rho)-A\left(f^{2} ; x, y ; \rho\right)\right]=f^{2}(x, y) \Delta \log f(x, y),
$$

which bears an important relation to the defining inequality (2) for smooth functions of class $P L$.

\section{BIBLIOGRAPHY}

1. E. F. Beckenbach and T. Radó, Subharmonic functions and surfaces of negative curvature, Trans. Amer. Math. Soc. vol. 35 (1933) pp. 662-674.

2. G. C. Evans, On potentials of positive mass, Trans. Amer. Math. Soc. vol. 37 (1935) pp. 226-253.

3. O. Frostman, Potentiel d'équilibre et capacité des ensembles . . , Thesis, Lund, 1935.

4. T. Rad6, Subharmonic functions, Berlin, 1937.

5. P. Reichelderfer and L. Ringenberg, The extension of rectangle functions, Duke Math. J. vol. 8 (1941) pp. 231-242.

6. F. Riesz, Sur les fonctions subharmoniques et leur rapport a la théorie du potentiel, Acta Math. vol. 54 (1930) pp. 321-360.

7. S. Saks, On the operators of Blaschke and Privaloff for subharmonic functions, Rec. Math. (Mat. Sbornik) N. S. vol. 9 (1941) pp. 451-456.

8. - - Theory of the integral, New York, 1937.

9. N. Wiener, Laplacians and continuous linear functionals, Acta Univ. Szeged. vol. 3 (1926) pp. 7-16.

Purdue University 